\title{
Planar modelling techniques for asymmetric building structures
}

\section{B. Stafford-Smith and M. Cruvellier}

\section{Dr D. Johnson, Nottingham Polytechnic}

The technique presented by the Authors allows asymmetric buildings to be modelled as plane frames; the advantage of using the model is that a standard analysis package may be employed without any program modifications. However, a more user-friendly approach would appear to be to incorporate the in-plane floor rigidity directly into the program. This can readily be achieved by designating one node on each floor level to be a master node, while the remaining nodes become slave nodes. If a master node is taken to be the origin of a Cartesian coordinate system, with the $x, y$-axes in the plane of the relevant floor, then the displacements in the floor plane $u, v$ and the vertical axis rotation $\theta$ of a slave node with coordinates $x$, $y$, may be related to the corresponding master quantities by the transformation

$$
\left[\begin{array}{l}
u \\
v \\
\theta
\end{array}\right]_{\mathrm{s}}=\left[\begin{array}{rrr}
1 & 0 & -y \\
0 & 1 & x \\
0 & 0 & 1
\end{array}\right]\left[\begin{array}{l}
u \\
v \\
\theta
\end{array}\right]_{\mathrm{m}}
$$

where subscript $\mathrm{s}$ indicates slave displacements and subscript $\mathrm{m}$ indicates master displacements.

24. By the use of this transformation, three degrees of freedom may be eliminated at each of the slave nodes, leaving a system with three degrees of freedom at each node, plus an extra three degrees of master freedoms at each floor level. This would be comparable with the degrees of freedom required by the planar model and is perhaps a more elegant solution. The rigid-diaphragm floor option, cited by the Authors, may, of course, operate in this fashion, in which case there would appear to be no reason why such diaphragms should not be employed with structures which incorporate membrane as well as beam elements.

\section{Professor Stafford-Smith and Mr Cruvellier}

The rigid floor option used by us in our reference three-dimensional analyses does indeed operate by way of the master-slave routine, described by Dr Johnson and by others previously. ${ }^{7}$ Although the rigid floor option is certainly 'user-friendly', it is not available in many structural analysis programs, and in some of those where it is offered, it may not be used with membrane elements. ${ }^{8}$

26. The purpose of the Paper, however, is not just to provide a technique for analysing three-dimensional structures by two-dimensional programs, but to show how a three-dimensional structure that twists as well as translates may be visualized as a two-dimensional model. In our opinion, our Paper provides a relatively 


\section{DISCUSSION}

simple solution to a problem that has been pursued over many years in increasingly esoteric and complex ways. It reduces the problem to one which requires a simple geometrical approach, rather than the more complex and less adequate, highly analytical approaches that have been proposed previously.

\section{References}

7. MacLeOD I. A. Structural analysis of wall systems. J. Instn Struct. Engrs, 1977, 55, No. 11, Nov., 487-495.

8. Wilson E. L. and Habibullah. SAP80, SAP90. Computers and Structures. Berkeley, California. 